\title{
Fine-Needle Aspiration Biopsy in the Diagnosis and Classification of Primary and Recurrent Lymphoma: A Retrospective Analysis of the Utility of Cytomorphology and Flow Cytometry
}

\author{
Henry Y. Dong, M.D., Ph.D., Nancy L. Harris, M.D., Frederic I. Preffer, Ph.D., Martha B. Pitman, M.D. \\ Department of Pathology, Massachusetts General Hospital, Harvard Medical School, Boston, \\ Massachusetts
}

We retrospectively reviewed our experience with the fine-needle aspiration biopsy (FNAB) diagnosis of primary and recurrent lymphoma to assess the ability of cytomorphology with and without ancillary flow cytometry (FCM) analysis to diagnose and subclassify these tumors according to the Revised European-American Lymphoma/World Health Organization classifications. We reviewed 139 consecutive FNABS of 84 primary and 55 recurrent lymphomas. FCM was successful in $105(75 \%)$ cases. The overall results, including cases without FCM, included 93/139 (67\%) true positive, $7(5 \%)$ false negative, and 39 indeterminate $(27$ [19\%] suspicious and 12 [9\%] atypical) diagnoses of lymphoma. In cases with FCM, there were 80/105 (77\%) true positive, no false negative, and 25 indeterminate diagnoses (15 [14\%] suspicious and 10 [9\%] atypical). The overall results of the 84 primary lymphomas were $55(67 \%)$ true positive, $5(5 \%)$ false negative, and 24 indeterminate $(14[16 \%]$ suspicious and 10 [12\%] atypical) diagnoses for lymphoma. Of the 68 primary lymphomas analyzed with FCM, 50 [74\%] were true positives, and 28 were indeterminate (11 [16\%] suspicious and $7[10 \%]$ atypical). There were no false negatives. Diagnostic accuracy varied among lymphoma subtypes. Subclassification of the positive cases were initially conclusive in only $55 / 93$ cases $(59 \%)$. However, a retrospective review of the morphologic together with FCM data in 15 of the 23 unclassified cases improved the overall subclassification of positive cases to $77 \%$. Subclassification was best in small lymphocytic lymphoma/chronic

Copyright ( $\odot 2001$ by The United States and Canadian Academy of Pathology, Inc.

VOL. 14, NO. 5, P. 472, 2001 Printed in the U.S.A.

Date of acceptance: January 4, 2001.

This work was presented in part as a poster presentation at the 87th annual meeting of the International Academy of Pathology (United States and Canadian divisions), February 28-March 6, 1998.

Address reprint requests to: Martha B. Pitman, M.D., Department of Pathology, Warren 2, Massachusetts General Hospital, 55 Fruit Street,

Boston, MA 02114; e-mail: mpitman@partners.org; fax: 617-726-7474. lymphocytic leukemia, lymphoplasmacytic lymphoma, Burkitt's lymphoma, mantle cell lymphoma, and plasmacytoma (all 100\%). Subclassification was poor in marginal-zone lymphoma (33\%), and initially as well in diffuse large B-cell lymphoma (62\%), but it improved on review (95\%), as did subclassification of follicular lymphoma (77 to $100 \%$ on review). Hodgkin's disease was recognized as malignant in only $44 \%$ of the cases $(7 / 16)$ and was classified as such based on morphology alone.This review of our early efforts to diagnose and subclassify lymphoma with FNAB and FCM indicates that although a diagnosis and proper subclassification of lymphoma can be made with certainty in the majority of cases, recurrent or primary, it requires close coordination of cytomorphology and immunophenotyping data, which often comes with close cooperation of cytopathologists and hematopathologists. A mere cytological diagnosis of positive for lymphoma is no longer acceptable if FNAB is to become an independent diagnostic tool for lymphoma.

KEY WORDS: Cytology, Diagnosis, Fine needle aspiration biopsy, Flow cytometry, Lymphoma, Subclassification.

Mod Pathol 2001;14(5):472-481

Fine-needle aspiration biopsy (FNAB) is a wellestablished tool for the diagnosis of malignant neoplasms, but its role in the diagnosis of lymphoma remains controversial. Although generally accepted for documenting recurrent lymphoma or for obtaining tissue for ancillary studies, such as immunological and molecular genetic analyses (1-21), FNAB for the primary diagnosis and subclassification of lymphoma in particular is less established (5-10). A cytological diagnosis of lymphoma on FNAB is still very often followed by a tissue biopsy in many cases, particularly for a primary diagnosis. Historically, this has been due in part to the fact that architectural pattern (nodular 
versus diffuse) was considered essential for lymphoma classification (22). Thus, a cytological diagnosis of "positive for lymphoma" has been generally acceptable because in recurrent lymphoma, this was sufficient for continued patient management, and in the case of a primary diagnosis, there was an expectation that an excisional biopsy would be performed for confirmation and subclassification. For FNAB to be accepted as an independent diagnostic test, however, this type of cytological diagnosis is not acceptable, because it does not provide sufficient information for clinicians to make therapeutic decisions (23).

With advances in the understanding of the biology, immunology, and molecular genetics of lymphoma in the past two decades, lymphoid neoplasms are now defined by a combination of morphologic, immunophenotypic, genotypic features, and characteristic clinical presentations. Architectural patterns, which historically have been the gold standard for lymphoma subclassification, are no longer of paramount importance in many cases. As a result, it should now be possible to diagnose and classify lymphoma subtypes that have a characteristic cytomorphology and immunophenotype without examination of tissue sections $(24,25)$.

Immunophenotyping is critical for lymphoma diagnosis and subclassification, particularly in the area of small-cell lymphomas, which have overlapping morphology both with reactive processes and with each other, making diagnosis and subclassification extremely difficult on cytomorphology alone (26-29). Immunophenotyping by flow cytometry (FCM) is a rapid and sensitive method to evaluate lymphoid markers (30). It appears to be especially suitable for cytology specimens because it requires only a small sample, is suitable for cells already in suspension, and has a rapid turnaround time. FCM gives quantitative results and can detect small abnormal cell populations in a reactive background; it can also assess co-expression of antigens on subsets of cells and the relative density of surface antigens. Although immunocytochemistry allows a close correlation between immunophenotype and cytomorphology (1-4, 31), FCM has the advantage of being able to identify a small population of $a b-$ normal cells that may not be apparent on morphology alone. This feature significantly improves the diagnostic sensitivity and thus is particularly helpful for the diagnosis of small B-cell lymphomas. In addition, current techniques allow detection of intracytoplasmic antigens, further closing the gap between application of FCM and immunohistochemistry.

In our hospital, FNAB for the diagnosis of lymphoma has been done with increasing frequency, but the use of FCM as well as the stringency of the approach to lymphoma subclassification have not been uniform in the past. To evaluate the role of
FCM and the feasibility for primary diagnosis and subclassification of lymphoma on FNAB, we undertook a retrospective review of our performance in this area. We retrospectively analyzed 139 consecutive cases of confirmed lymphoma during a period when FNAB was gradually adapted as a routine diagnostic tool to evaluate lymphoid lesions at our institution. Our aims were to assess the ability to diagnose lymphoma using a combination of FNAB/ FCM in nodal and extranodal sites, the ability to subclassify lymphoma with these techniques, the applicability of such an approach as an independent diagnostic test for the diagnosis of primary lymphoma, and the limitations of the technique.

\section{MATERIALS AND METHODS}

\section{Case Selection}

FNABs performed at the Massachusetts General Hospital during a 2-and-a-half-year period (January 1995 to July 1997) were reviewed retrospectively when FNAB was evolving as an independent diagnostic test for the diagnosis of lymphoma in our hospital. Cases were identified through a computer search of the department database for lymphoid lesions. These cases initially included all FNAB from lymph nodes and from various extranodal sites with a prominent lymphoid component on the smears. Many of the cases, particularly the extranodal sites, were performed for a mass not clinically suspected of being a lymphoma. Only 139 FNAB from 129 patients in whom a definitive diagnosis of lymphoma was ultimately made were chosen for this study, excluding benign and reactive lesions, as well as metastatic nonlymphoid malignancies. Aspirates with insufficient tissue for diagnosis were also excluded. The final diagnosis of lymphoma was made by a combination of cytology, flow cytometry immunophenotyping, surgical or core biopsy, and in certain recurrent cases, with clinical information alone. There were 95 patients corresponding to 102 FNAB who eventually had surgical biopsies done, either needle core or excisional biopsies. On 37 FNAB from 34 patients, a diagnosis of lymphoma (12 primary) was made by FNAB \pm FCM without a subsequent tissue biopsy.

Primary lymphoma was defined as a previously undiagnosed lymphoma in which the FNAB was the primary diagnostic test. Recurrent lymphoma was defined as one in which the diagnosis of lymphoma had been made by a previous biopsy procedure.

\section{FNAB Specimen Processing}

The FNAB specimens were obtained by either a cytopathologist or radiologist using a 22-25-gauge needle with multiple passes. Direct smears were 
made and stained with a rapid hematoxylin and eosin stain, a standard Papanicolaou stain, or an air-dried Romanowsky-type stain. It was routine to attempt to obtain aspirated tissue for FCM analysis on all FNAB that demonstrated a significant lymphoid component on the rapid interpretation smears. Usually, additional dedicated aspirates were performed solely to obtain cells for FCM. This was not accomplished or possible in all cases, however. In addition, some cases proved insufficient for FCM because of scant cellularity, cellular degeneration, and excessive peripheral blood contamination.

For deep-seated lesions in which lymphoma was detected on rapid interpretation, core biopsies were procured and sent to the frozen section lab as a back-up for immunohistochemistry, should the aspirate for flow cytometry be insufficient.

\section{Diagnostic Data}

To analyze our actual performance over this time interval, we used for this study the diagnoses issued in the cytopathology and flow cytometry reports. For all cases that were not subclassified on either report and for which a tissue biopsy had provided a definitive subclassification, the slides were retrieved and reviewed by MBP and HYD, together with the flow cytometry data, but without knowledge of the final diagnosis.

In all cases, cytopathologists performed the evaluation of the cytomorphology and issued the primary report; hematopathologists performed the evaluation of the flow-cytometric analysis and issued an addendum report on each case. Needle rinsings from each aspirate, and in most cases, dedicated aspirates just for needle rinsing in saline, were used for FCM analysis. When available on radiologically guided biopsies, tissue fragments from supplemental needle core biopsies were frozen for histologic and immunohistologic examination. During this time period, there was no standardized approach to the coordination of flow cytometry and cytomorphology for a final diagnosis, particularly with respect to subclassification. In rare cases, the cytopathology report was issued without knowledge of FCM results, for example, in obviously malignant cases when subclassification was not attempted, whereas in others, these results were known and necessary for defining clonality. The cytopathology reports used general headings of positive, suspicious, or negative for malignant lymphoma or were atypical with a descriptive diagnosis.

\section{Flow Cytometry}

All cytology specimens containing a dominant lymphoid component were submitted for FCM.
Cases with limited available tissue, those suspicious for Hodgkin's lymphoma, and some of the recurrent lymphomas were not submitted for FCM. Four-color flow-cytometric analysis was done according to standard procedures (30) on a FACScan (Becton-Dickenson, San Diego, CA), and data were analyzed with the Lysys software (BectonDickenson). The antibody panels routinely used included CD19, CD20, CD5, CD10, CD23, CD3, CD7, CD4, CD8, CD43, CD45, CD14, and antibodies to immunoglobulin light chains (kappa and lambda). In addition to analysis of surface antigens, cells were also permeabilized for intracytoplasmic staining of immunoglobulin light chains or CD3 when indicated. A diagnosis of lymphoma was made if there was a B-cell population with a restricted (monotypic) light chain, or a dominant T-cell population with aberrant phenotype or phenotypes (lack and/or abnormal distribution of one or more panT-cell antigens). An absolute kappa to lambda lightchain ratio of $>6$ or $<0.3$ was usually required. However, the final interpretation also depended on other abnormalities, such as B cells expressing dim CD20 or coexpressing CD10 or CD5. A case was considered suspicious for lymphoma if analysis detected a borderline light-chain excess or a small subset of monotypic B cells or abnormal T cells. A negative diagnosis was made if only polyclonal $\mathrm{B}$ cells and/or normal T cells were found. FCM from cases with too few cells for analysis or only necrotic material was excluded.

\section{Definitions of Diagnosis and Classification Used}

A case was classified as "positive for lymphoma" in this study only if a positive diagnosis was definitively made by either cytomorphology or FCM, or both. A specimen that was diagnosed as suspicious for lymphoma by both morphology and FCM remained suspicious rather than positive. All cases were classified according to the REAL/WHO lymphoma classification system $(24,25)$. A case was considered to have been subclassified on FNAB when the final report indicated that the results were diagnostic of, or consistent with, a particular lymphoma subtype on either the cytology or FCM report. Cases signed out as "positive for lymphoma," "high-grade lymphoma," "low-grade lymphoma," or "small-cell lymphoma" were not considered to have been subclassified. Subclassification of Hodgkin's disease was not attempted.

\section{RESULTS}

One hundred thirty-nine FNAB from 129 patients were analyzed in this study, including 120 patients who had a single FNAB, 8 patients who had two FNAB and 1 patient who had three. In patients with 
more than one FNAB, the aspirates were all performed at different times and/or from different lesions. Nodal sites constituted 56\% (78/139) and extranodal sites $44 \%$ (61/139), the majority of which were obtained from the head and neck region, some of which likely represent lymph nodes. FCM was successfully performed on 105 (76\%) cases. Overall, there were $84(60 \%)$ cases representing a primary diagnosis of malignant lymphoma and 55 $(40 \%)$ cases of recurrent lymphoma.

The final results taken from cytology and/or flow cytometry reports are summarized in Table 1 . Of all cases, 67\% (93/139) were diagnosed as "positive for lymphoma," $19 \%$ (27/139) were diagnosed as suspicious, and 9\% (12/139) were considered atypical, warranting further evaluation and a subsequent biopsy. Overall, 5\% (7/139) of cases were falsely negative. The positive rate was similar between cases of primary and recurrent lymphoma $(66 \%$ versus $69 \%)$.

There were $105(76 \%)$ cases with adequate tissue for FCM analysis. The overall positive rate for a diagnosis of lymphoma by FCM was $77 \%$ with a false negative rate of zero. Similarly, the positive rate for the diagnosis of primary lymphoma was $74 \%$. Among five cases considered cytologically negative because of a polymorphous lymphoid component, one was monoclonal by FCM analysis and subsequently confirmed to be an extranodal marginal-zone B-cell lymphoma (MZL). In addition, 3 of 18 cytologically suspicious cases and 2 of 12 cytologically atypical cases were positive for lymphoma by FCM. There were 17 cases of lymphoma in which FCM was negative. Ten of these were Hodgkin's disease (HD). The others included one anaplastic large-cell lymphoma (ALCL), one follicle center lymphoma (FL), one MZL, two plasmacytomas, and two diffuse large B-cell lymphomas (DLBCL).

\section{Accuracy of Diagnosis among Lymphoma Subtypes}

Analysis of the different lymphoma subtypes showed variation in the frequency with which they were positively identified as lymphoma. Overall, the positive diagnosis rate was $100 \%$ for B-cell chronic

TABLE 1. Summary of General Cytology Diagnoses With or Without Flow Cytometry (FCM) Analysis

\begin{tabular}{|c|c|c|c|c|}
\hline \multirow{2}{*}{ Diagnosis } & \multicolumn{2}{|c|}{ All cases, $n(\%)$} & \multicolumn{2}{|c|}{$\begin{array}{c}\text { Primary Cases, } n \\
(\%)\end{array}$} \\
\hline & $\pm \mathrm{FCM}$ & $+\mathrm{FCM}$ & $\pm \mathrm{FCM}$ & $+\mathrm{FCM}$ \\
\hline Positive & $93(67)$ & $80(77)$ & $55(66)$ & $50(74)$ \\
\hline Suspicious & 27 (19) & $15(14)$ & $14(16)$ & $11(16)$ \\
\hline Atypical & $12(9)$ & $10(9)$ & $10(12)$ & $7(10)$ \\
\hline Negative & $7(5)$ & 0 & $5(6)$ & 0 \\
\hline Total & 139 & 105 & 84 & 68 \\
\hline
\end{tabular}

lymphocytic leukemia/small lymphocytic lymphoma (B-CLL/SLL; 9/9), Burkitt's lymphoma (BL; $2 / 2$ ), mantle cell lymphoma (MCL; $2 / 2)$, ALCL (2/2), and plasmacytoma (5/5; Table 2). Three of four cases of lymphoplasmacytic lymphoma (LPL; 75\%), 26/34 cases of DLBCL (76\%), 15/22 FL (68\%), and $3 / 5(60 \%)$ peripheral T-cell lymphoma (PTCL) were also recognized as lymphoma. However, only 7/15 MZL (47\%) and 7/16 HD (44\%) were identified by either morphology or FCM as malignant.

When only 105 cases with FCM are considered, 3/3 LPL (100\%), 21/24 DLBCL (88\%), 13/18 FL (72\%), and $3 / 3$ PTCL (100\%) were recognized as lymphoma in addition to CLL/SLL, MCL and BL. For the B-cell lymphomas, the diagnosis rested on detecting monotypic immunoglobulin light-chain expression; the three PTCL were recognized as cytologically malignant $\mathrm{T}$ cells with or without aberrant $\mathrm{T}$-cell antigen expression. However, even with FCM, only 7/ 15 (47\%) MZL were initially recognized definitely as neoplastic, having a distinct monotypic population by FCM. Six additional cases had a small monotypic population or a slight light-chain excess, which was not felt to be diagnostic of malignancy, and they lacked sufficient cytologic atypia to be recognized as malignant on morphologic grounds alone. All were diagnosed as suspicious or atypical, leading to additional biopsies.

\section{Subclassification of Lymphoma}

Of the 93 cases diagnosed as positive for lymphoma, 55 were subclassified on the initial reports (59\%; Table 2). On review of 15 of the 26 unclassified but potentially classifiable cases, 12 additional cases were subclassified (total, 72\%). Excluding 23 "unclassifiable" cases ( $16 \%$ of total), which were excluded because of an inability to confirm the subtype on subsequent biopsy, the $68 \%$ initial accuracy was improved to $83 \%$ after review. When only classifiable cases with FCM were considered (excluding HD and ALCL for which FCM is not beneficial), the accuracy rate was $74 \%$ in the initial reports, increasing to $92 \%$ after review (Table 2).

The accuracy of subclassification varied among the subtypes. Among the non-Hodgkin's lymphomas, all cases of SLL/CLL (Fig. 1A, C), LPL, MCL (Fig. 1B-C), BL, plasmacytoma and PTCL, on which FCM was done, were correctly classified in the initial reports. FL was correctly classified initially in 10 of 15 cases (67\%; Fig. 2A-C); on review, three additional cases with FCM were correctly subclassified ( $87 \%$ of all cases and $100 \%$ of cases with FCM). Two cases of FL without FCM could not be subclassified. One case each of MCL and plasmacytoma that lacked FCM and one case of ALCL (Fig. 3C) with normal FCM findings were recognized as lymphoma but not subclassified. 
TABLE 2. Subclassification of 139 Fine-Needle Aspiration Biopsy Cases of Lymphoma With or Without Flow Cytometry Analysis Before and After Review of Unclassified Cases

\begin{tabular}{|c|c|c|c|c|c|c|c|}
\hline Lymphoma & $\begin{array}{r}\text { Total } \\
\text { Cases }\end{array}$ & $\begin{array}{c}\text { Positive- } \\
\text { all Cases, } \\
\text { n (\%) }\end{array}$ & $\begin{array}{l}\text { Subclassified in } \\
\text { report-all } \\
\text { cases, } \mathrm{n}(\%)\end{array}$ & $\begin{array}{c}\text { Subclassified } \\
\text { after review- } \\
\text { All Cases, n (\%) }\end{array}$ & $\begin{array}{l}\text { Positive- } \\
\text { with FCM, } \\
\text { n (\%) }\end{array}$ & $\begin{array}{l}\text { Subclassified in } \\
\text { Report-with } \\
\text { FCM, n (\%) }\end{array}$ & $\begin{array}{c}\text { Subclassified after } \\
\text { review with FCM, } \\
\text { n (\%) }\end{array}$ \\
\hline $\mathrm{HD}$ & 16 & $7(44)$ & 7 (100) & 7 (100) & N/A & N/A & N/A \\
\hline SLL/CLL & 9 & $9(100)$ & $9(100)$ & $9(100)$ & $9 / 9$ & $9(100)$ & 9 (100) \\
\hline LPL & 4 & $3(75)$ & $3(100)$ & $3(100)$ & $3 / 3$ & $3(100)$ & $3(100)$ \\
\hline Plasmacytoma & 5 & $5(100)$ & $4(80)$ & $5(100)$ & $4 / 4$ & $3(75)$ & $4(100)$ \\
\hline MZL & 15 & 7 (47) & $1(14)$ & $2(28)^{a}$ & $6 / 13(46)$ & $1(17)$ & $2(33)$ \\
\hline FL & 22 & $15(68)$ & $10(67)$ & $13(87)^{\mathrm{b}}$ & $13 / 15(87)$ & $10(77)$ & $13(100)$ \\
\hline MCL & 2 & $2(100)$ & $1(50)$ & $1(50)$ & $1 / 1$ & $1(100)$ & $1(100)$ \\
\hline DLBCL & 34 & $26(76)$ & $14(54)$ & $21(81)^{\mathrm{c}}$ & $21 / 24(88)$ & $13(62)$ & $20(95)$ \\
\hline $\mathrm{BL}$ & 2 & 2 (100) & 2 (100) & 2 (100) & $2 / 2$ & 2 (100) & $2(100)$ \\
\hline PTCL & 5 & $3(60)$ & $3(100)$ & $3(100)$ & $3 / 4(75)$ & $3(100)$ & $3(100)$ \\
\hline ALCL & 2 & 2 (100) & $1(50)$ & $1(50)$ & N/A & N/A & N/A \\
\hline Subtotal & 116 & $81(70)$ & 55 (68) & 67 (83) & $62 / 78(80)$ & $45 / 62(73)$ & $57 / 62(92)$ \\
\hline Unclassifiable & 23 & $12(52)$ & & & & & \\
\hline TOTAL & 139 & $93(67)$ & 55 (59) & $67(72)$ & $74 / 90(82)$ & $45 / 74(61)$ & $57 / 74(77)$ \\
\hline
\end{tabular}

a Slides not available for review on $4 / 6$ unclassified cases.

b Slides not available for review on $2 / 5$ unclassified cases.

c Slides not available for review on $3 / 12$ unclassified cases.

HD, Hodgkin's disease; SLL, small-lymphocytic lymphoma; CLL, chronic lymphocytic lymphoma; LPL, lymphoplasmacytic lymphoma; MZL, marginal-zone B-cell lymphoma; FL, follicle-center lymphoma; MCL, mantle cell lymphoma; DLBCL, diffuse large B-cell lymphomas; BL, Burkitt's lymphoma; PTCL, peripheral T-cell lymphoma; ALCL, anaplastic large-cell lymphoma.

Of the 26 cases of DLBCL (Fig. 3A), only 14 were classified on the initial report (54\%); the remainder were diagnosed as lymphoma on the cytology report and recognized as a B-cell lymphoma on the FCM report. Slides were available for review in 9/12 of these unclassified DLBCL cases, and 7 could be correctly subclassified simply by commenting on the large-cell morphology and/or characters of forward light scatter, increasing the subclassification rate to $81 \%$. Two contained an admixture of smaller cells and could not be confidently subclassified.

MZL (Fig. 3B) proved most difficult to subclassify, with only one of seven (14\%) cases subclassified on the initial report. Slides were available for review on only two of six unclassified cases; one of these could be subclassified on review, for an overall accuracy of $28 \%$, or $33 \%$ considering only those cases with FCM.

Seven of 16 HD (Fig. 3D) were recognized as malignant, and all were correctly classified on cytomorphology alone. The remaining nine cases were considered either atypical or suspicious for HD but lacked diagnostic Reed-Sternberg cells, thus leading to subsequent biopsy.

In summary, of the 26 cases that were not subclassified on FNAB/FCM initially and for which a tissue biopsy had provided definitive classification, slides were available for review in 15 cases. These included 2/6 MZL, 9/12 DLBCL, and 3/5 FL and 1/1 plasmacytoma. Of these, 12 cases could be further subclassified using morphology and FCM data, including 1/2 MZL, 7 /9 DLBCL (large cells), 3/5 FL $(\mathrm{CD} 10+)$, and 1 anaplastic plasmacytoma (CD45-, CD38+, CD56+). One case of MZL could not be subclassified even with FCM, and two cases of DLBCL had admixed small cells and could not be subclassified. For another two cases (one MCL, one ALCL), slides were not reviewed because of lack of FCM analysis, for they would be unlikely to be subclassified based on morphology alone.

There were 23 cases of NHL that were unclassifiable by cytology and FCM in the initial reports, only 12 of which were positive for lymphoma by morphology and/or FCM. Of these cases, 13 had no subsequent biopsy (all recurrent cases without available history of previous classification), and 10 had only a core biopsy, on which a definite subclassification was not possible. Many had very limited clinical information. Further analysis demonstrated that all 12 cases were composed of CD19+, CD20+ $B$ cells with variable cell size, 3 were $\mathrm{CD} 10+$, and all were CD5-. Thus, it is plausible that they included three additional FL, and the reminder were likely to be DLBCL, FL (CD10-), or MZL (see discussion).

\section{DISCUSSION}

We have evaluated a series of 139 confirmed lymphomas diagnosed using FNAB with and without FCM immunophenotyping analysis, including 84 cases $(60 \%)$ in which this represented the primary diagnosis of lymphoma. We compared our diagnostic accuracy for cases with FCM with that of all cases, evaluating both the ability to positively diagnose lymphoma and the frequency with which cases were accurately subclassified. For a small number of initially unclassified but potentially subclassifiable cases, the available slides and FCM data were reviewed and cases subclassified if possible.

The ability to recognize lymphoma by FNAB is well established (1-21). However, controversy still 


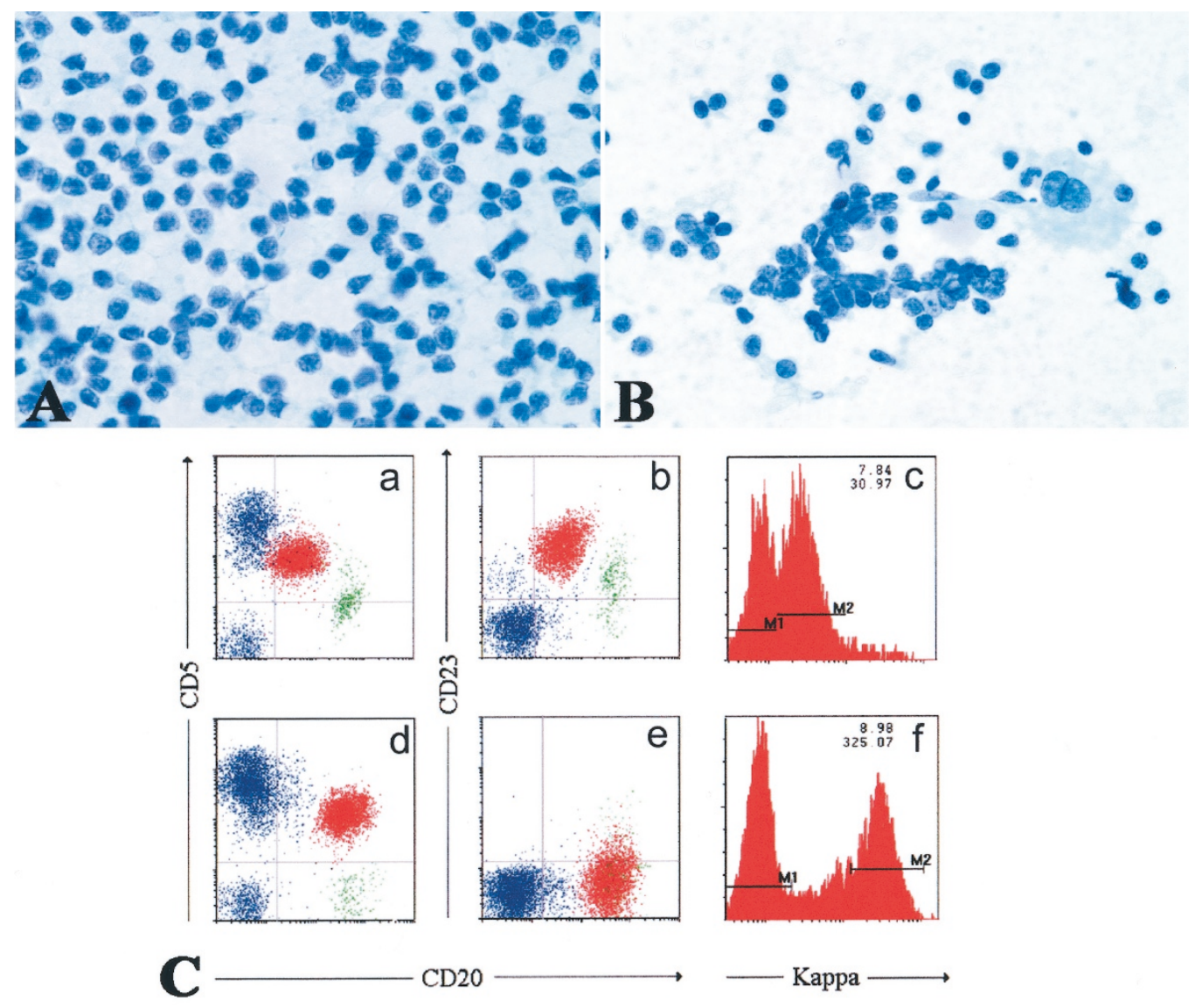

FIGURE 1. A, Chronic lymphocytic leukemia (CLL)/small lymphocytic lymphoma (SLL). Cytology smears show a monotonous small, round lymphocyte population with round, noncleaved nuclei (Papanicolaou stain; $900 \times$ ). B, Mantle cell lymphoma. Cytology smears show a monotonous small lymphocyte population with irregular nuclear contours, nuclear grooves, the absence of transformed large lymphoid cells, and the occasional large epitheliod histiocyte (Papanicolaou stain; $900 \times$ ). C, A comparison of the flow cytometry between these two lymphoma subtypes shows that the cells of CLL/SLL $(a-c)$ coexpress CD23 and CD5 with dim CD20 and dim monotypic surface IgL (kappa in this case), whereas the cells of MCL ( $d-f$ ) coexpress CD20 and CD5 without CD23 and demonstrate bright monotypic surface light chain (kappa in this case).

remains that centers on the ability of FNAB to replace excisional biopsy for a primary diagnosis and subclassification of lymphoma. Cytomorphology coupled with FCM analysis provided a definitive diagnosis of lymphoma in $77 \%$ of the cases in our series (82\% of non-Hodgkin's lymphomas). The accuracy rate was similar for primary and recurrent lymphomas. Without FCM, the accuracy rate was $67 \%$, supporting the importance of immunophenotyping as an important adjunct to the cytological diagnosis and subclassification of lymphoma. Those cases with FCM not considered cytologically malignant were all either cytologically suspicious or atypical, leading to further evaluation or biopsy. As such, with the routine use of FCM for the evaluation of all lymphoid dominant lesions, our falsenegative rate was zero.

Our overall diagnostic rate is similar to those of previous studies $(4-10,20)$ indicating that collectively, FNAB combined with FCM is applicable in routine practice to the diagnosis of lymphoma, both recurrent and primary tumors. Meda et al. (4), in a recent series, found cytology and FCM able to correctly diagnose and subclassify lymphoma in $77 \%(158 / 206)$ of NHL, including $72 \%(86 / 119)$ of primary cases. They especially emphasized the importance of close coordination between morphology and immunophenotyping for accurate diagnosis and subclassification. The value of such a coordinated effort is illustrated by the improved overall subclassification rate in this series from 73 to $92 \%$ among positive cases.

Dunphy and Remos (8) reported their results of FNAB and FCM in 73 cases of clinically suspected lymphoma. A positive diagnosis was made in 36/45 $(80 \%)$ of recurrent lymphoma cases and 10/14 (71\%) of primary cases. Cases suspicious or equivocal for lymphoma, which would often comprise a significant portion of cases in practice, were not mentioned, and subclassification was not attempted in this series. Young et al. (9) recently reported their experience with $100 \mathrm{FNAB}$, most of which were combined with results of FCM. Of the 81 cases of confirmed lymphoma, $58(72 \%)$ cases were diagnosed cytologically as positive, 18 (22\%) as suspicious, and $5(6 \%)$ as equivocal. Although the 

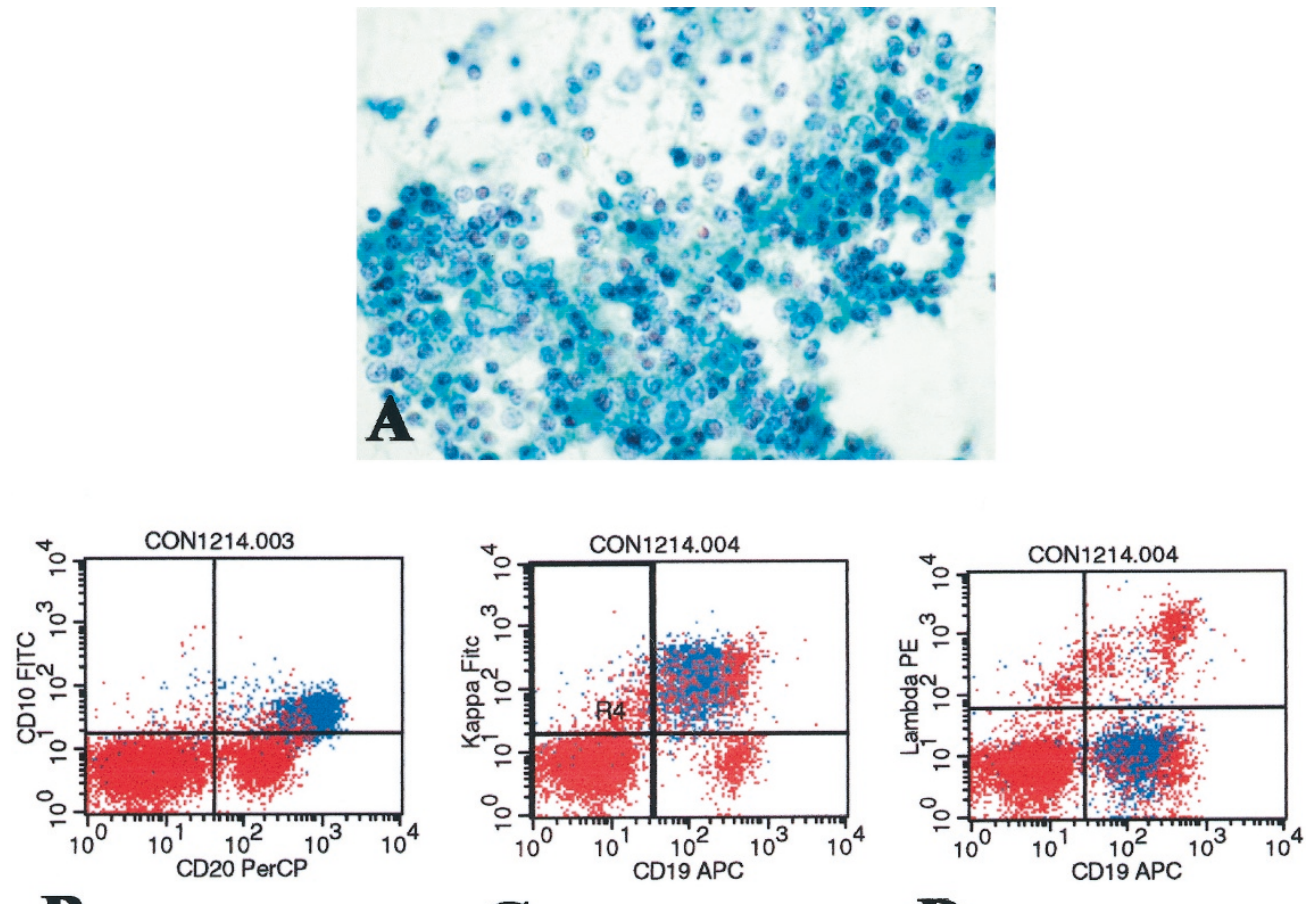

B

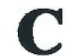

D

FIGURE 2. A, Follicular lymphoma. A slightly dispersed follicle demonstrates a polymorphous appearing population of slightly atypical lymphoid cells (Papanicolaou stain; 560×). B-D, Flow cytometry shows that the tumor cells (blue), although present in a reactive lymphoid background (red), are B cells (CD20) that coexpress CD10 (B) and are light-chain restricted: kappa positive (C) and lambda negative (D).
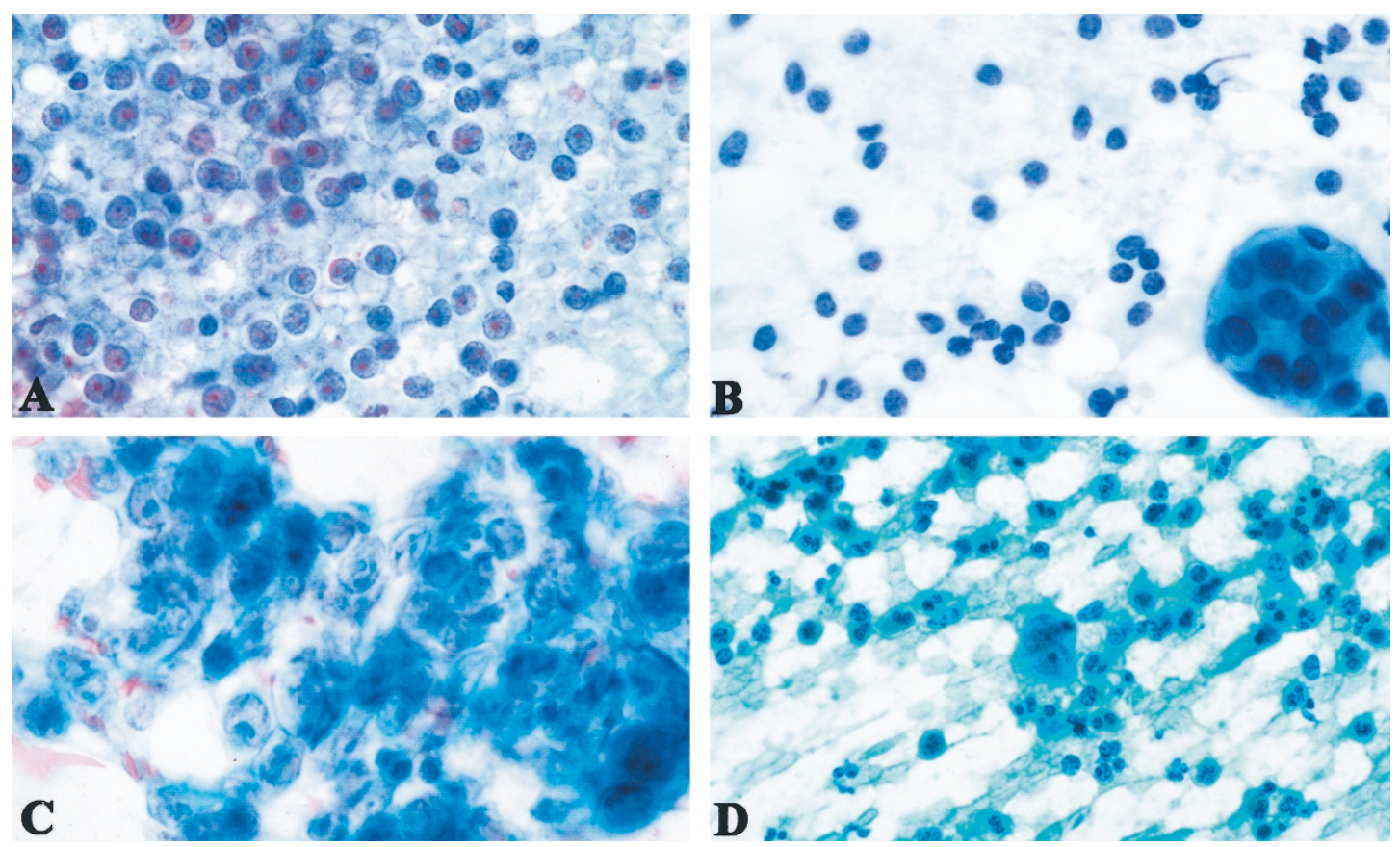

FIGURE 3. A, Diffuse large, B-cell lymphoma. Cytology smears show a dispersed population of large, malignant lymphoid cells with clumped chromatin, prominent nuclei, and variably stripped cytoplasm (Papanicolaou stain; 900×). B, Marginal-zone lymphoma. In this extranodal example, cytology smears demonstrate salivary gland acini (lower left) associated with a polymorphous population of small, only slightly atypical lymphoid cells. Marginal-zone cells, characterized by a rim of clear cytoplasm, are visible but may be difficult to appreciate in wet fixed preparations (Papanicolaou stain; 900×). C, Anaplastic large-cell lymphoma. Cytology smears show large, anaplastic tumor cells with prominent nuclei in a cohesive cluster, a feature that can cause confusion with non-hematopoietic malignancies (Papanicolaou stain; 900×). D, Hodgkin's disease. Cytology smears that demonstrate typical Reed-Sternberg cells are diagnostic (Papanicolaou stain; $560 \times$ ). 
number of positive cases for each lymphoma subtype was listed, the feasibility of subclassification by FNAB/FCM was not discussed. Sneige et al. (7) reported on 220 FNAB, a majority of which had a lymphoma diagnosis before the FNAB. Among them, 173/220 (79\%) were positively diagnosed as lymphoma by FNAB in conjunction with immunocytochemistry. Another 15 cases $7 \%$ of the total cases) were inconclusive, of which 14/15 were proven to be lymphoma on subsequent biopsies, and 23 benign lesions were interpreted as reactive. Like ours, none of these studies had any true false positive diagnoses.

One of the main limitations to FNAB as an independent diagnostic tool for lymphoma has been the inability to subclassify cases for directing appropriate therapy, given the reliance of previous classifications on architectural features. The REAL/WHO classification has incorporated new criteria independent of tissue architecture in the diagnosis and subclassification of lymphoma. Thus, diseases with characteristic immunophenotypes and/or cytomorphology should be classifiable with FNAB. We found that lymphoma subtypes with characteristic morphology and specific immunophenotypic profiles were in fact diagnosed and subclassified with high accuracy, including B-CLL/SLL, BL, MCL, LPL and plasmacytoma. For example: B-CLL/SLL is characterized by a monomorphic small lymphoid cell population with round nuclei, clumped chromatin, and occasional nucleoli that displays a B-cell phenotype $(\mathrm{CD} 19 / 20+)$ co-expressed with CD5 and CD23 without CD10. Monotypic light-chain and CD20 expression are dim (Fig. 1, A, C). Neoplastic cells in BL are medium sized, monomorphic cells with round nuclei, multiple nucleoli, and abundant basophilic vacuolated cytoplasm that are associated with tingible body macrophages, and they demonstrate an immunophenotype of CD19/ $20+, \mathrm{CD} 43+$ and CD10+ but are CD5- and TdT- . In contrast, most cases of FL are composed of CD10+, CD43- small B cells in a reactive background (Fig. 3). MCL is composed of a monotonous small lymphoid-cell population with irregular nuclear membranes, grooves, and inconspicuous nucleoli, with scant to no cytoplasm that displays a CD19/20+, CD5+, CD23-, and CD10- immunophenotype with moderate monotypic light chain and CD20 expression (Fig. 1, B-C). LPL is characterized by a small lymphoid cell population with plasmacytoid differentiation of varying degrees. Although the immunophenotype is relatively nonspecific, detection of monotypic intracytoplasmic light chain is a common finding among these cases, and when combined with the cytomorphology, allows for proper subclassification. Plasmacytoma is a tumor generally characterized by readily recognized plasma cells that immunophenotypically express bright CD38 and a cytoplasmic monotypic light chain with absence of CD19 and CD45. However, occasional cases with a high-grade morphology can cause confusion with large cell/immunoblastic lymphoma, as in one of our cases. Not all subtypes of lymphomas exhibit specific features, thus, the challenge remains for the classification of tumors with nonspecific and/or overlapping features. We found that the initial cytopathology and/or FCM reports had accurately subclassified only $54 \%$ of DLBCL, $67 \%$ of FL, and $14 \%$ of MZL.

In cases of DLBCL, the malignant morphologic features are typically so apparent that FCM analysis is often not necessary for a confident morphological diagnosis of lymphoma. As such, anticipating a subsequent biopsy for confirmation and subclassification, many of our earlier cases were simply diagnosed as "positive for lymphoma." Thus, 26/34 cases $(76 \%)$ of DLBCL were recognized as lymphoma with certainty on FNAB/FCM, but only $59 \%$ of these positive cases (14/26) were initially subclassified as DLBCL. The simple mention of the large-cell predominance on smears or the recognition of cell size as indicated by FCM light-scatter analysis improves the classification rate as evidenced by the improved subclassification rate of $81 \%$ (95\% when only considering those cases with FCM) on review of 7/12 unclassified cases. Interestingly, it was largely recognition of cell size that allowed for subclassification of most of the 46 cases described by Meda et al. (4) (36/46 [76\%] recognized as large-cell lymphoma). FCM analysis of DLBCL in their series was polyclonal or falsely negative in 16 cases, mostly attributable to a limited sample for FCM analysis. We did not experience this with our cases. One potential pitfall we did encounter was the difficulty in the distinction of some cases from Grade $3 \mathrm{FL}$. There were three cases of proven DLBCL that were CD10+, indicating a follicle center cell origin. Fortunately, a patient with either high-grade FL or DLBCL would be treated similarly.

MZL and FCL are two lymphomas with heterogeneous morphologic features. MZL is also characterized by a nonspecific immunophenotype. Thus, they were more difficult to subclassify than other lymphomas, even retrospectively. FL and MZL both have morphologic features that overlap with a benign reactive process (26-28), being composed of a variably mixed population of small atypical cells with irregular nuclei and rare large cells. Monocytoid B cells, the characteristic cell of MZL, with abundant clear cytoplasm, may not be easy to recognize on smears, especially on wet fixed tissue. In addition, the high content of reactive cells may impair detection of a monoclonal B-cell population. Although CD10 expression in a small B-cell lymphoma is likely to be specific for a diagnosis of 
$\mathrm{FL}$, it is not positive in all cases and is reported to be positive on only about $60 \%$ of the cases by immunohistochemistry or $90 \%$ by FCM $(4,32-34)$. Moreover, CD10 is also expressed on cells of BL and some cases of DLBCL, causing diagnostic difficulty in the distinction from Grade 2-3/3 FL.

Of a total of $22 \mathrm{FL}$ cases in our series, only 15 (68\%) could be diagnosed as lymphoma in initial reports. Among them, 10/15 (67\%) were initially subclassified as FL because of expression of CD10. On review of three of the five initially unclassified cases, three additional were found to have $\mathrm{CD} 10+$ small B cells, improving the subclassification of FL in this series to $87 \%$, or to $100 \%$ when considering only those cases with FCM.

MZL often lacks specific cytologic features, although in occasional cases, a prominent monocytoid appearance can be appreciated. Of 15 total cases of MZL, fewer than half were initially reported as lymphoma $(6 / 15 ; 47 \%)$. On review, one additional case was classified as positive and subclassified as MZL. All seven positive cases of MZL had monotypic light-chain restriction, but only four were cytologically malignant. In addition, small monotypic populations were found in additional six cases of MZL by FCM. Even though these six cases did not completely fulfill the criteria required in this study for defining a positive diagnosis, they were highly suspicious for lymphoma and clearly warranted further evaluation. Our results are in agreement with other reports that MZL is difficult to diagnose and subclassify by FNAB even after extensive ancillary studies (26-28).

In summary, our retrospective analysis showed a relatively high diagnostic accuracy for FNAB in the diagnosis of primary and recurrent lymphoma (67\%), which was substantially improved with the addition of flow cytometry $(77 \%)$. We found that continued use of the traditional cytopathology diagnostic category of "positive for lymphoma" frequently obscured the correct subclassification of some lymphomas. This was particularly true for MZL and DLBCL, which may lack diagnostic immunophenotypes and require careful integration of cytomorphology and immunophenotype for correct subclassification. When we reviewed the original material, we were able to correctly subclassify $72 \%$ of the cases overall, and $77 \%$ of those with flow cytometry-an accuracy rate for primary diagnosis that certainly seems to validate the procedure and justify its use as a primary diagnostic tool with lymphomas. The two lymphomas with the lowest diagnostic accuracy were HD and MZL. Although HD could be correctly recognized as malignant and could be classified by cytomorphology alone in half the cases, flow cytometry was of no added value except in excluding non-Hodgkin's lymphoma. Because distinction between classical and lymphocyte predominance HD remains important in determining treatment and prognosis and often requires immunophenotyping, either immunocytochemistry on smears or open biopsies will be needed in most cases of HD. The diagnosis of MZL requires integration of cytomorphology, immunophenotype, and clinical features, and until the morphologic and clinical spectrum of this disease is better understood, many cases may continue to need open biopsies for diagnosis and classification.

An issue that was not dealt with in this study but that needs further consideration is the availability of material for cytogenetic or molecular genetic analysis for diagnosis and of archival material for correlative scientific studies. Although it is clear that immunophenotyping by either flow cytometry or immunocytochemistry is essential for the diagnosis of lymphoma on FNAB, cytogenetic studies are not considered routine in lymphoma diagnosis. Many lymphomas have characteristic cytogenetic abnormalities that can be important in diagnosis or prognosis, including SLL/CLL, MCL, MZL, FL, and BL. Material obtained by FNAB is ideal for routine cytogenetic studies, and consideration should be given to submitting needle rinsing for cytogenetic analysis or making additional smears for FISH analysis in cases in which a rapid interpretation suggests one of these lymphomas.

When open biopsies are performed for diagnosis, there is always an archive of paraffin-embedded tissue-and often frozen tissue - that can be used for diagnostic molecular genetic analysis, additional immunophenotyping, or research. One concern about the increasing use of FNAB for primary diagnosis and classification of lymphomas is the potential loss of archival tissue for research. This is particularly acute in the present era of emerging technologies of genomics and proteomics. Although many laboratories prepare additional smears that can be stored for future studies, the suitability of these for molecular genetic analysis is questionable. For academic centers with a commitment to diagnostic FNAB, consideration should be given to establishing banks of viably frozen cells. These could be used for diagnostic molecular genetic analysis should a definite diagnosis not be made on routine morphology and immunophenotyping and could also serve as an archive of material for future immunophenotyping and genetic studies.

Acknowledgments: We thank David Beech and Barrett Goodspeed for their help in collecting data and Ivona Oszak and Abby Kelliher for assisting the collection of flow cytometry data. Additional thanks go to Steve Connely and Michelle Forrestall for their assistance in the production of the illustrations. 


\section{REFERENCES}

1. Levitt S, Cheng L, Du Puis M, Layfield L. Fine needle aspiration diagnosis of malignant lymphoma with confirmation by immunoperoxidase staining. Acta Cytol 1985;29:895-902.

2. Oertel J, Oertel B, Kastner M, Lobeck H, Huhn D. The value of immunocytochemical staining in lymph node aspirates in diagnostic cytology. Br J Haematol 1988;70:307-16.

3. Tani EM, Christensson B, Porwit A, Skoog L. Immunocytochemical analysis and cytomorphological diagnosis on fine needle aspirates of lymphoproliferative diseases. Acta Cytol 1988;32:209-15.

4. Meda BA, Buss DH, Woodruff RD, Cappellari JO, Rainer RO, Powell BL, et al. Diagnosis and subclassification of primary and recurrent lymphoma. The usefulness and limitations of combined fine-needle aspiration cytomorphology and flow cytometry. Am J Clin Pathol 2000;113:688-99.

5. Cafferty LL, Katz RL, Ordonez NG, Carrasco CH, Cabanillas FR. Fine needle aspiration diagnosis of intraabdominal and retroperitoneal lymphomas by a morphologic and immunocytochemical approach. Cancer 1990;65:72-7.

6. Sneige N, Dekmezian R, el-Naggar A, Manning J. Cytomorphologic, immunocytochemical, and nucleic acid flow cytometric study of 50 lymph nodes by fine-needle aspiration. Comparison with results obtained by subsequent excisional biopsy. Cancer 1991;67:1003-7.

7. Sneige N, Dekmezian RH, Katz RL, Fanning TV, Lukeman JL, Ordonez NF, et al. Morphologic and immunocytochemical evaluation of 220 fine needle aspirates of malignant lymphoma and lymphoid hyperplasia. Acta Cytol 1990;34:31122.

8. Dunphy C, Ramos R. Combining fine-needle aspiration and flow cytometric immunophenotyping in evaluation of nodal and extranodal sites for possible lymphoma: a retrospective review. Diagn Cytopathol 1997;16:200-6.

9. Young NA, Al-Saleem TI, Ehya H, Smith MR. Utilization of fine-needle aspiration cytology and flow cytometry in the diagnosis and subclassification of primary and recurrent lymphoma. Cancer 1998;84:252-61.

10. Johnson A, Akerman M, Cavallin-Stahl SE. Flow cytometric detection of B-clonal excess in fine needle aspirates for enhanced diagnostic accuracy in non-Hodgkin's lymphoma in adults. Histopathology 1987;11:581-90.

11. Frable WJ, Kardos TF. Fine needle aspiration biopsy. Applications in the diagnosis of lymphoproliferative diseases. Am J Surg Pathol 1988;12(1 Suppl):62-72.

12. Chernoff WG, Lampe HB, Cramer H, Banerjee D. The potential clinical impact of the fine needle aspiration/flow cytometric diagnosis of malignant lymphoma. J Otolaryngol 1992;1:1-15.

13. Katz RL, Wojcik EM, el-Naggar AK, Ordonez NG, Johnston DA. Proliferation markers in non-Hodgkin's lymphoma. A comparative study between cytophotometric quantitation of Ki-67 and flow cytometric proliferation index on fine needle aspirates. Anal Quant Cytol Histol 1993;15:179-86.

14. Moriarty AT, Wiersema L, Snyder W, Kotylo PK, McCloskey DW. Immunophenotyping of cytologic specimens by flow cytometry. Diagn Cytopathol 1993;9:252-8.

15. Hanson CA. Fine needle aspiration and immunophenotyping. A role in diagnostic hematopathology? [editorial]. Am J Clin Pathol 1994;101:555-6.

16. Saleh H, Masood S. Value of ancillary studies in fine-needle aspiration biopsy. Diagn Cytopathol 1995;13:310-5.

17. Leong AS, Stevens M. Fine-needle aspiration biopsy for the diagnosis of lymphoma: a perspective. Diagn Cytopathol 1996;15:352-7.
18. Katz RL. Controversy in fine needle aspiration of lymph nodes. A territorial imperative? [editorial]. Am J Clin Pathol 1997;108(1 Suppl):S3-5.

19. Jeffers MD, McCorriston J, Farquharson MA, Stewart CJ, Mutch AF. Analysis of clonality in cytologic material using the polymerase chain reaction (PCR). Cytopathology 1997;8: $114-21$.

20. Lui K, Mann KP, Vitellas KM, Paulson EK, Nelson RC, Gockerman JP, et al. Fine-needle aspiration with flow cytometry immunophenotyping for primary diagnosis of intraabdominal lymphomas. Diagn Cytopathol 1999;21:98-104.

21. Vianello F, Tison T, Radossi P, Poletti A, Galligioni A, Giacon C, et al. Detection of B-cell monoclonality in fine needle aspiration by PCR analysis. Leuk Lymphoma 1998;29:17985.

22. The Non-Hodgkin's Lymphoma Pathologic Classification Project. National Cancer Institute sponsored study of classification of non-Hodgkin's lymphomas: summary and description of a working formulation for clinical usage. Cancer 1982;49:2112-35.

23. Wakely PE Jr. Fine-needle aspiration cytopathology in diagnosis and classification of malignant lymphoma. Accurate and reliable? Diagn Cytopathol 2000;22:120-5.

24. Harris HL, Jaffe ES, Stein H, Banks PM, Chan JK, Cleary ML, et al. A revised European-American classification of lymphoid neoplasms: a proposal from the International lymphoma Study Group. Blood 1994;84:1361-92.

25. Harris NL, Jaffe ES, Diebold J, Flandrin G, Muller-Hermelink HK, Vardiman J, et al. The World Health Organization Classification of Hematological Malignancies. Report of the Clinical Advisory Committee Meeting-Airlie House, Virginia, November 1997. Mod Pathol 2000;13:193-207.

26. Puttagunta L, Harris NL, Pitman MB. The cytologic diagnosis of marginal zone B cell lymphoma: a comparative analysis with other low grade B cell lymphoma [Abstract]. Mod Pathol 1998;11:42A

27. McNeely TB. Diagnosis of follicular lymphoma by fine needle aspiration biopsy. Acta Cytol 1992;36(6):866-8.

28. Cha I, Long SR, Ljung BM, Miller TR. Low-grade lymphoma of mucosa-associated tissue in the parotid gland: a case report of fine-needle aspiration cytology diagnosis using flow cytometric immunophenotyping. Diagn Cytopathol 1997;16:345-9.

29. Stewart CJ, Duncan JA, Farquharson M, Richmond J. Fine needle aspiration cytology diagnosis of malignant lymphoma and reactive lymphoid hyperplasia. J Clin Pathol 1998;51:197-203.

30. Preffer FI. Flow cytometry. In: Colvin RB, Bhan AK, McCluskey RT, editors. Diagnostic immunopathology. 2nd ed. New York: Raven Press; 1993. p. 725-49.

31. Robins DB, Katz RL, Swan F Jr, Atkinson EN, Ordonez NG, Huh YO. Immunotyping of lymphoma by fine-needle aspiration. A comparative study of cytospin preparations and flow cytometry [see comments]. Am J Clin Pathol 1994;101: 569-76.

32. Almasri NM, Iturraspe JA, Braylan RC. CD10 expression in follicular lymphoma and large cell lymphoma is different from that reactive lymph node follicles. Arch Pathol Lab Med 1998;122:539-44.

33. Chu PG, Chang KL, Arber DA, Weiss LM. Immunophenotyping of hematopoietic neoplasms. Semin Diag Pathol 2000; 17:236-56.

34. Ferry JA, Harris NL. Atlas of lymphoid hyperplasia and lymphoma. Philadelphia: Saunders; 1997. p. 87. 